\title{
Sepsis neonatal: aspectos fisiopatológicos y biomarcadores
}

\author{
José S. Cortés* \\ Laura X. Fernández Cruz** \\ Emilce Beltrán Zúñiga**** \\ Carlos F. Narváez $z^{* * * *}$ \\ Carlos Eduardo Fonseca-Becerra*****
}

\begin{abstract}
* Médico en servicio social obligatorio. Laboratorio de Infección e Inmunidad. Facultad de Salud. Universidad Surcolombiana. Neiva. Huila. Colombia. **Médico Pediatra. Servicio de Pediatría. Hospital Universitario de Neiva. Neiva. Huila. Colombia.

***Médico Pediatra. Servicio de Pediatría. Hospital Susana López de Valencia. Popayán. Cauca. Colombia.

****Médico. Doctor en Inmunología. Programa de Medicina. Facultad de Salud. Universidad Surcolombiana. Neiva. Huila. Colombia.

*****édico Pediatra. Programa de Medicina. Facultad de Salud. Universidad Surcolombiana. Unidad de Cuidado Intensivo Neonatal, Hospital Universitario de Neiva. Neiva. Huila. Colombia.

Correspondencia: Dr. José S. Cortés. Dirección: Calle 9 No 14-02. Neiva. Colombia. Correo electrónico: jsancg@gmail.com
\end{abstract}

\section{Resumen}

La sepsis neonatal es una causa importante de morbilidad y mortalidad en recién nacidos a nivel mundial. Su diagnóstico es difícil por sus manifestaciones clínicas inespecíficas y la poca disponibilidad de métodos diagnósticos eficientes. En la fisiopatología de la sepsis se ha descrito una respuesta inmune excesiva o suprimida que puede conducir a desenlaces potencialmente fatales. Se ha estudiado la utilidad pronóstica, diagnóstica y de seguimiento de factores solubles que se alteran en la sepsis neonatal y se han agrupado bajo el término biomarcadores de sepsis neonatal. Aquí se describen los principios fisiopatológicos de la sepsis neonatal y las características de los biomarcadores más usados para su diagnóstico, además, se mencionan detalles de otros marcadores que también han sido estudiados recientemente. Actualmente, se recomienda el uso de un biomarcador temprano en combinación con uno tardío para lograr un mejor rendimiento, sin embargo, aún no se ha identificado un biomarcador ideal para la sepsis neonatal. MÉD.UIS.2019;32(3):35-47

Palabras clave: Recién nacido. Sepsis Neonatal. Interleucina-6. Proteína C Reactiva. Procalcitonina. Biomarcadores.

\section{Neonatal sepsis: physiopathology and biomarkers}

\section{Abstract}

Neonatal sepsis is a major cause of morbidity and mortality in newborns worldwide. Its diagnosis remains a challenge due to the nonspecific clinical findings and the lack of efficient diagnostic tools. In the physiopathology of neonatal sepsis, an excessive or suppressed immune response has been described, which can lead to potentially fatal conditions. The prognostic, diagnostic, and follow-up value of several soluble factors altered in neonatal sepsis has been studied. These have been grouped under the term neonatal sepsis biomarkers. Here, aspects of the physiopathology in neonatal sepsis and the characteristics of the most studied biomarkers used for neonatal sepsis diagnosis are described, also, details about other recently studied markers are mentioned. Currently, the use of an early-warning biomarker together with a late-warning biomarker is recommended to get higher diagnostic accuracy. However, a single ideal biomarker for neonatal sepsis has not been found yet. MÉD.UIS.2019;32(3):35-47

Keywords: Newborn. Neonatal sepsis. Interleukin-6. C-reactive protein. Procalcitonin. Biomarkers.

¿Cómo citar este artículo?: Cortés JS, Fernández LX, Beltrán E, Narváez CF, Fonseca-Becerra CE. Sepsis neonatal: aspectos fisiopatológicos y biomarcadores. MÉD.UIS.2019;32(3):35-47. doi: 10.18273/revmed.v32n2-2019005 


\section{Introducción}

La sepsis neonatal (SN) es una infección sistémica de etiología bacteriana, viral, parasitaria o fúngica ${ }^{1,2}$, asociada a disfunción orgánica que pone en riesgo la vida de los recién nacidos $(\mathrm{RN})^{3}$.

En 2016, El Tercer Consenso Internacional para la Definición de Sepsis y Choque Séptico (Sepsis-3)4, definió la sepsis como una disfunción orgánica que amenaza la vida, causada por una desregulación de la respuesta del hospedero frente a una infección. En los neonatos, la sepsis se clasifica dependiendo del momento de su inicio: SN temprana es la que inicia durante las primeras $72 \mathrm{~h}$ de vida, caso en el que la infección, como una infección placentaria o del tracto genital materno, es transmitida de manera vertical al RN5. Si la infección inicia desde las 72 h hasta los 28 días de vida, se clasifica como SN tardía ${ }^{6}$; la infección en este último grupo de pacientes es transmitida desde el ambiente hospitalario o la comunidad 5 . Esta clasificación implica entonces diferencias en el modo de trasmisión posible de la infección, así como los posibles agentes etiológicos, por lo cual es de utilidad para decidir el tratamiento antibiótico inicial7.

El riesgo de $\mathrm{SN}$ y la mortalidad tienen una relación inversa con la edad gestacional y el peso al nacer, ya que en estas condiciones se encuentra un sistema inmune inmaduro, los pacientes están más expuestos a medidas como ventilación mecánica, hospitalización prolongada, inserción de línea central y otras medidas invasivas 5 . Factores maternos como corioamnionitis, la ruptura prematura de membranas y niveles elevados de Proteína $C$ reactiva $(P C R)$ maternos también son factores de riesgo conocidos para SN temprana ${ }^{8,9}$.

La SN es la causa más importante de morbilidad y mortalidad en el RN, tanto pretérmino como a término5. Según la Organización Mundial de la Salud, cerca de cinco millones de muertes anuales se deben a la $\mathrm{SN}^{10}$. A pesar de la importancia a nivel mundial, el interés público en la sepsis es pobre aún ${ }^{4}$. La mayoría de casos de SN a nivel mundial tienen su origen en infecciones respiratorias o gastrointestinales ${ }^{11}$. El S. agalactiae o Streptococcus del grupo B (SCB) de Lancefield es el principal microorganismo asociado a $\mathrm{SN}$ temprana en el mundo ${ }^{12}$. Cerca del $70 \%$ de casos de SN tardía son por microorganismos grampositivos ${ }^{7}$. En los Estados Unidos predominan las cepas de
SBG y los microorganismos gramnegativos ${ }^{13}$. Allí, la incidencia de SN temprana está alrededor de 0.30.98 por 1000 nacidos vivos ${ }^{14,15}$, con una mortalidad cerca del $24.4 \%{ }^{16}$. Actualmente, existe tendencia al aislamiento de Staphylococcus spp. en SN tardía y neonatos sometidos a procedimientos invasivos ${ }^{13}$. En el Reino Unido, la incidencia de SN temprana es de 0.9 casos por 1000 nacidos vivos ${ }^{17}$. En los países en vía de desarrollo, la incidencia de SN varía entre 1-4 eventos por 1000 nacidos vivos ${ }^{18}$. En Egipto se ha reportado que hasta el 50\% de los pacientes de UCIN presentan SN ${ }^{19}$. En Sudáfrica, se han reportado tasas de fatalidad para la SN temprana y tardía de hasta $40 \%$ y $19.7 \%$, respectivamente, cifras similares a las de otros países en desarrollo ${ }^{10}$. La incidencia de SN reportada en México es de 160.9 casos por cada 1000 nacidos vivos ${ }^{13}$, en América del Sur y el Caribe las cifras estuvieron entre 3.59-8.91 casos por cada 1000 nacidos vivos para el $2005^{13}$. En Colombia en el año 2014, se registraron 7.24 muertes neonatales por 1000 nacidos vivos ${ }^{20}$. Un estudio realizado en la ciudad de Medellín, en el año 2008, evidenció que cerca de $7 \%$ de los pacientes hospitalizados en UCIN correspondían a pacientes con $\mathrm{SN}^{21}$. Los principales gérmenes aislados fueron los Staphylococcus coagulasa negativa y los bacilos gramnegativos ${ }^{21}$.

Las manifestaciones clínicas de la $\mathrm{SN}$ no son específicas e incluyen rechazo al alimento, distrés respiratorio, neumonía, apnea, retraso del llenado capilar, frialdad de extremidades, intolerancia a la alimentación, inestabilidad térmica, hipotonía, convulsiones, abultamiento de la fontanela e ictericia prolongada7. Las manifestaciones más severas son el choque, la coagulación intravascular diseminada, falla multiorgánica, entre otras ${ }^{5,22}$. Estos hallazgos se pueden encontrar en muchas otras condiciones patológicas lo que dificulta el diagnóstico ${ }^{5}$, sumado a que también pueden presentarse de manera tardía, empeorando el pronóstico para el RN55.

El diagnóstico final de SN se hace por hemocultivo, considerado como el estándar de oro, del que se pueden obtener resultados en un periodo de $36 \mathrm{~h}^{17}$ hasta los 6 días $^{14}$ de cultivo. Aunque regularmente sólo son positivos en $30-70 \%$ de los $\operatorname{casos}^{14,17}$, por lo que no se puede descartar SN al obtener un resultado negativo. En parte, el bajo rendimiento de los hemocultivos se debe a un volumen insuficiente de muestra, variaciones en los niveles de bacteremia, uso de antibióticos en la madre o previo a la toma de la 


\section{Septiembre - Diciembre}

muestra en el neonato, entre otros ${ }^{23}$. Recientemente se ha demostrado la utilidad de sistemas multiplex basados en reacción en cadena de polimerasa para la detección rápida de infecciones nosocomiales en RN pretérmino, con buena sensibilidad y especificidad, lo que podría servir como prueba diagnóstica adjunta al estándar de oro $^{24}$. La iniciación de terapia antibiótica antes de obtener los resultados del cultivo se recomienda para los neonatos con signos clínicos o factores epidemiológicos asociados con sepsis ${ }^{10}$.

El tratamiento antibiótico resulta en muchas ocasiones como consecuencia del temor a una infección neonatal ${ }^{17}$, sus graves complicaciones y secuelas a largo plazo ${ }^{14}$, se ha reportado que en el 80$95 \%{ }^{14}$ de RN tratados con antibióticos por sospecha de infección, no requerían su uso ${ }^{17}$.

Teniendo en cuenta que dentro de la fisiopatología de la SN, después del reconocimiento de una señal que puede provenir de un agente patógeno o señales de daño endógenas, se presenta un aumento en la producción de varias clases de mediadores inflamatorios y anti-inflamatorios ${ }^{25}$, como los reactantes de fase aguda, interleucinas y otras moléculas. Se ha planteado que estos mediadores podrían servir como biomarcadores de $\mathrm{SN}$ y que podrían ser de ayuda para establecer su diagnóstico, ayudando así con la disminución del uso innecesario de antibióticos. Así mismo, se ha planteado que los biomarcadores podrían ayudar a predecir la severidad de la SN y a guiar el tratamiento antibiótico ${ }^{25,26}$.

En estudios previos se ha analizado el uso de diversos parámetros como ayuda en el diagnóstico de SN como índices hematológicos ${ }^{10,27,28}$, biomarcadores como la PCR, la procalcitonina (PCT), citocinas como la interleucina (IL)-1 $\beta$, IL-8, IL-6, interferón (IFN)- $\gamma$, factor de necrosis tumoral (TNF)- $\alpha$, quimiocinas como CXCR4 y CXCL12 ${ }^{6}$, marcadores de superficie celular, niveles de ácidos nucleicos en suero o plasma ${ }^{29}$.

En esta revisión se describen los principios fisiopatológicos de la sepsis neonatal y las características de los biomarcadores más usados para su diagnóstico. Además, se mencionan detalles de otros marcadores que también han sido recientemente estudiados.
Sepsis neonatal: aspectos fisiopatológicos y biomarcadores

\section{Metodología de búsqueda}

Desde junio de 2017 hasta diciembre de 2018, por medio del motor de búsqueda PUBMED se buscaron y revisaron artículos científicos, usando las palabras clave "neonatal sepsis", "biomarkers", "c reactive protein", "procalcitonin", "interleukin 6", "immunology" y "pathogenesis". Se incluyeron los artículos publicados en los últimos cinco años y de investigación en humanos. En esta búsqueda fueron identificados 38 artículos, con base en el título y el resumen. De estos, fueron excluidos cinco por ser duplicados. También se usó como referencia el Tercer Consenso Internacional para la Definición de Sepsis y Choque Séptico (Sepsis-3) ${ }^{4}$ como fuente de la definición de sepsis, dos artículos acerca de la inmunopatogenia de SN y cuatro artículos sobre epidemiología de $\mathrm{SN}$ que no fueron identificados durante la búsqueda, pero que los autores consideraron que, por su relevancia en el tema, era pertinente incluirlos en la presente revisión. Se revisaron en total 40 artículos para esta revisión.

\section{Resultados}

\section{Principios fisiopatogénicos de la sepsis neonatal}

Gran parte de la susceptibilidad, frecuencia y severidad de la sepsis neonatal se debe a las características del sistema inmune propias de la edad, explicado por importantes diferencias entre la inmunidad en neonatos y personas adultas ${ }^{1,30}$. Debido al tiempo que necesita la maduración del sistema inmune adaptativo, los mecanismos de defensa en los neonatos se fundamentan en el sistema inmune innato ${ }^{30}$. Sin embargo, los RN pretérmino con menos de 32 semanas de edad gestacional tienen su sistema inmune innato también inmaduro ${ }^{11}$, lo que los hace aún más vulnerables frente a procesos infecciosos ${ }^{11}$. De hecho, hasta el $25 \%$ de los RN prematuros con menos de 32 semanas de gestación desarrollan una infección sistémica severa dentro de su periodo neonatal"1.

El sistema inmune inmaduro de los RN muestra una serie de diferencias cuantitativas y cualitativas en comparación con los individuos de mayor edad ${ }^{31}$, que van a determinar las respuestas frente a estímulos patógenos (Ver Figura 1). 


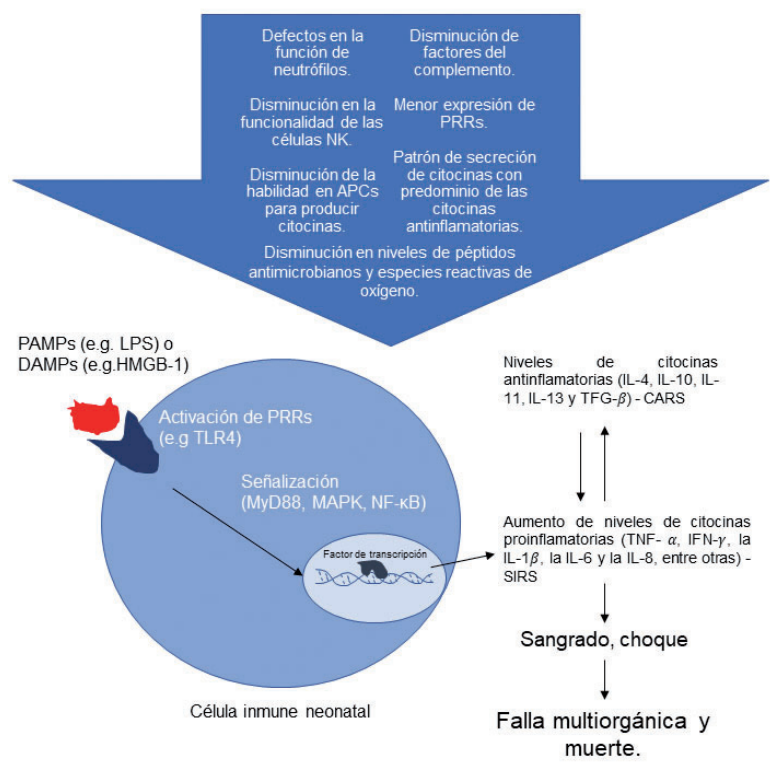

Figura 1. Particularidades del sistema inmune que afectan la inmunopatogénesis de la sepsis neonatal

PRRs: receptores de reconocimiento de patrones. APCs: células presentadoras de antígenos. PAMPs: patrones moleculares asociados a patógenos. DAPMs: patrones moleculares asociados a daño. LPS: lipopolisacárido. HMGB-1: proteínas de alta movilidad del grupo de caja 1

Fuente: autores

La frecuencia de linfocitos $T$ y $B$ circulantes diferenciados a células de memoria y efectoras solo alcanzan hasta el $10 \%$ del total de linfocitos del $\mathrm{RN}^{11}$. Los neutrófilos representan una cifra mínima de los leucocitos circulantes antes de las 32 semanas de gestación, además, tienen defectos en el reclutamiento, la actividad fagocítica, la expresión de moléculas de adhesión ${ }^{2}$ y liberación de péptidos antimicrobianos y especies reactivas de oxígeno (ROS). Adicionalmente, los RN pretérmino carecen de la habilidad de formar redes extracelulares y péptidos antimicrobianos que eliminan microorganismos mediante las especies reactivas de oxígeno ${ }^{11,31}$; limitaciones en el aclaramiento de estos componentes facilita la diseminación sistémica y el agravamiento del proceso séptico ${ }^{29}$. Los factores del sistema del complemento como C3a, Factor H, Factor I también están disminuidos en los RN pretérmino, lo que limita el reconocimiento, opsonización y aclaramiento de patógenos ${ }^{11}$. El plasma neonatal tiene una disminución importante en la actividad opsonizadora, comparada con los adultos, lo que incrementa el riesgo de sepsis². Hay tres subtipos descritos de monocitos, según la expresión de CD14 y CD16 en su superficie; antes de las 29 semanas de gestación, la expresión de CD14 es baja"1 lo que limita la función de reconocimiento inmune y presentación antigénica de estas células"1. También, por debajo de las 24 semanas de gestación, poca actividad de los receptores de reconocimiento de patrones (PRR, en inglés) es detectada. La actividad de los PRR incrementa significativamente hasta la semana 33 de gestación, cuando alcanza un nivel similar al del RN a término"1. Inicialmente se produce la activación de los PRR endosomales, como los receptores Toll-Like (TLR, en inglés) 7, 8, y 9, luego los intracitoplasmáticos, como los receptores de dominio de oligomerización por unión de nucleótidos (NOD, en inglés), y los receptores NOD-like (NLR, en inglés), seguidos de los PRR extracelulares, como los TLR 1, 2, 4, y $5^{11}$. La actividad del TLR 2, con un rol importante en el reconocimiento de Staphylococcus coagulasa negativa, se desarrolla tarde en la gestación ${ }^{11}$. La respuesta de Th mediada por IL-17 parece ser importante en prevenir la invasión de microorganismos a través de las mucosas y por microorganismos intracelulares ${ }^{11,31}$. Con el fin de promover esta respuesta, las células presentadoras de antígenos neonatales producen grandes cantidades de IL-1 $\beta$, IL-6, y de IL-23, no obstante, son producidas en bajos niveles en los RN pretérmino de menos de 29 semanas de gestación. En los neonatos, las funciones de las células NK, tales como la desgranulación, la liberación de IFN- $\gamma$ y factores citolíticos están disminuidas en comparación con las respuestas que se producen en individuos mayores ${ }^{31}$. También se conoce que las poblaciones de células dendríticas neonatales, tanto convencionales como plasmocitoides, muestran una disminución en la habilidad de producir múltiples citocinas asociado a la activación por medio de los TLR ${ }^{31}$.

La flora microbiana del útero, la placenta, el canal vaginal y la piel cercana se modifica cerca al nacimiento. En el caso de los RN a término, hay factores que promueven la colonización por microorganismos benéficos ${ }^{11}$. Este microbioma tiene un papel importante en la protección del RN contra infecciones ${ }^{11}$. Los RN a término que nacen por vía vaginal son colonizados por la flora cutánea, intestinal y vaginal de la madre ${ }^{11}$. En el caso de los RN pretérmino, más de la mitad nacen por cesárea, lo que probablemente altera la colonización normal, además, frecuentemente presentan algún déficit nutricional o están expuestos a tratamientos antibióticos prolongados, lo que puede alterar aún más la colonización normal y aumenta el riesgo de $\mathrm{SN}^{11}$. Estas alteraciones de la flora normal favorecen el crecimiento de su contraparte patógena ${ }^{11}$. 


\section{Septiembre - Diciembre}

Los gérmenes aislados en la SN temprana están relacionados con la flora presente en la madre, adquirida al momento del parto o durante la gestación?. La SN tardía puede ser causada por la entrada sistémica de bacterias ambientales vía catéter o por traslocación a través del intestino inmaduro, especialmente cuando ha sido comprometido, como en el caso de enterocolitis necrotizante ${ }^{29}$. El reconocimiento de los patógenos por células inmunes locales es el primer paso para el desarrollo de una respuesta inmune una vez que se ha comprometido la barrera epitelial ${ }^{2}$.

Las células inmunes reconocen patógenos intra y extracelulares por medio de estructuras microbianas características, o patrones moleculares asociados a patógenos (PAMP, en inglés), mediante los PRR. Los PRR más estudiados han sido los TLR. Hay 10 tipos de TLR en humanos, cada uno con ligandos específicos $^{2}$. El lipopolisacárido (LPS, o endotoxina) de las bacterias gramnegativas es el PAMP más estudiado, y es reconocido principalmente por el TLR 4 y su vía de señalización ${ }^{31}$, de la que hacen parte las proteínas de membrana CD14 y $\mathrm{MDz}^{2}$. Otro PAMP bien conocido es el ácido lipoteicoico, de las bacterias grampositivas, el cual es reconocido por medio del TLR $2^{2}$. Los RNA virales de doble cadena hacen su señalización por medio del TLR 3, un receptor intracelular ${ }^{2}$. Frecuentemente, un microrganismo estimula más de un tipo de TLR simultáneamente ${ }^{2}$.

Otros PRR intracelulares son los receptores NOD y los receptores NOD-like, que detectan peptidoglicanos de las bacterias grampositivas en el citosol, los receptores de lectinas tipo C, los receptores del Gen I inducible por ácido retinoico (RIG-I, en inglés) y los receptores RIG-like (RIR, en inglés), los cuales detectan RNA viral de doble cadena e inducen la producción de IFN-12,11. La activación de los PRR activa una cascada de señalización, en la que intervienen múltiples factores como el factor de diferenciación mieloide 88 (MyD88), el factor regulador de interferones 5 (IRF5, más relacionado con la vía de producción de interferones), que llevan finalmente a la activación de las proteínas quinasas activadas por mitógenos (MAPK) y del regulador de transcripción maestro NF-kB, lo que a su vez lleva a la expresión genética y producción de citocinas proinflamatorias ${ }^{2,11,16,32}$. La señalización por medio de STAT6 lleva a la estimulación de la producción de citocinas antinflamatorias" ${ }^{11}$. Las citocinas son moléculas pequeñas con una corta vida media, que va desde minutos hasta algunas horas, y tienen un papel central en la migración y activación de las células inmunes ${ }^{25}$.

Recientemente, se ha señalado la importancia de los patrones moleculares asociados a daño (DAMP, en inglés), que son proteínas intracelulares que se liberan como respuesta a un estímulo nocivo, pueden también estimular los TLR, y además amplifican la respuesta de receptores como el receptor para los productos finales de la glicación (RAGE, en inglés), lo que perpetúa la respuesta inflamatoria debido a una traslocación de DAMP de la madre al feto en el contexto de $\mathrm{SN}^{25}$. Las proteínas de alta movilidad del grupo de caja 1 (en inglés, HMGB-1) son DAMP importantes que señalizan por medio de los TLR 2, TLR 4 y por RAGE, y se han relacionado con la progresión de la sepsis a choque séptico². La sepsis se asocia con disregulación en la muerte celular, incluyendo autofagia, apoptosis y piroptosis', llevando a la liberación de DAMP que estimulan aún más la respuesta inflamatoria ${ }^{3}$. Las proteínas de choque térmico, otros DAMP conocidos, también señalizan a través de algunos TLR².

Actualmente es aceptado que la patogénesis de la SN se caracteriza por una respuesta inmune bimodal excesiva o suprimida, cuyas dos fases pueden ocurrir simultáneamente ${ }^{30}$. El inicio de este proceso se da entonces como respuesta al reconocimiento por los PRR de los componentes de los organismos invasores, lo cual estimula la liberación de una variedad de citocinas ${ }^{25}$. EI TNF- $\alpha$, IFN- $\gamma$, la IL-1 $\beta$, la IL-6 y la IL-8 son citocinas proinflamatorias, abundantes en la primera fase de la SN, conocida como síndrome de respuesta inflamatoria sistémica (SIRS, en inglés)². La estimulación de los neutrófilos, plaquetas, células endoteliales y otras células inmunes llevan a la producción de mediadores biológicamente activos como el factor de activación plaquetaria, metabolitos del ácido araquidónico, histamina, bradicinina, proteínas del complemento, péptidos vasoactivos y óxido nitroso, mediadores proinflamatorios que contribuyen con la respuesta inflamatoria sistémica característica del SIRS ${ }^{25}$. Se cree que el incremento moderado de estas citocinas proinflamatorias tiene un rol protector al promover las respuestas antimicrobianas, mientras que una elevación excesiva se asocia con lo que se conoce como tormenta de citocinas, se asocia con un desenlace fatal debido a fallas multiorgánicas ${ }^{30}$. La IL-10, IL-11, IL-13 y el factor de crecimiento transformante (TFG)- $\beta$ son citocinas anti-inflamatorias ${ }^{2}$, producidas en la segunda fase de la respuesta inmune, conocida como síndrome de 
respuesta anti-inflamatoria compensadora (CARS, en inglés), especialmente en los pacientes con sepsis severa ${ }^{25}$. Estas citocinas antinflamatorias bloquean la activación de los fagocitos, la fiebre, alteran la coagulación y disminuyen los niveles de mediadores vasoactivos. Los diferentes patrones de incremento de las citocinas pueden ayudar en la identificación del factor etiológico ${ }^{30}$.

La disfunción inmune se relaciona con la progresión de la sepsis, debido a la disfunción de subpoblaciones de células $T$, incluyendo las células T reguladoras, cuya activación puede inhibir el exceso en la respuesta inflamatoria del huésped debido a la activación de las vías del $T C R^{22}$. Otras células con funciones inmunosupresoras son las células $B$ reguladoras y las células dendríticas reguladoras ${ }^{22}$. Las células $\mathrm{B}$ reguladoras ejercen su función inmunosupresora por la secreción de IL-10, mientras que las células $T$ reguladoras pueden hacerlo también mediante inhibición por contacto al unir moléculas expresadas en su membrana, como el CTLA-4, TIM-3, y GITR, a su receptor en las células efectoras ${ }^{22}$.

El estrés oxidativo que resulta de la respuesta del huésped frente a las endotoxinas de los microorganismos gramnegativos y a las exotoxinas de los grampositivos es responsable de la activación de radicales libres y citocinas proinflamatorias 33 . Aunque los neonatos en el contexto de una infección tienen mayor actividad de enzimas antioxidantes como la superóxido dismutasa y la glutatión peroxidasa, en comparación con los adultos, los neonatos tienen menores niveles de agentes antioxidantes como vitamina $\mathrm{E}, \beta$-carotenos y grupos sulfihidrilo, por lo cual, frente a un desbalance oxidativo y proinflamatorio, ocurre un significativo aumento en los niveles de $P C R^{33}$.

Las alteraciones en la coagulación ocurren en casi todos los pacientes sépticos ${ }^{28}$. Durante un proceso infeccioso, la cascada de la coagulación empieza con el aumento de la expresión de factor tisular en los neutrófilos, monocitos o el endotelio, lo que lleva a un aumento de proteínas de la coagulación incluyendo el complejo trombina antitrombina, el inhibidor del activador del plasminógeno y el complejo plasmina- $a 2$ antiplasmina ${ }^{2}$. Además, también hay una inactivación de la proteína $\mathrm{S}$ y otras proteínas anticoagulantes, como la antitrombina $\mathrm{III}^{2}$. Estos desórdenes de la coagulación que se presentan en la sepsis pueden ir desde hipercoagulabilidad localizada, lo que es en realidad un mecanismo de defensa que tiene como fin limitar la infección, pero que puede llevar a tromboembolismo y hasta coagulación intravascular diseminada (CID), caracterizada por la presencia de trombosis microvascular ${ }^{2}$. En el caso de sepsis por gramnegativos, es el LPS lo que induce la activación de tromboplastina tisular que promueve el desarrollo de $\mathrm{CID}^{3}$. Luego de la aparición de CID, los desórdenes hemorrágicos se presentan debido al agotamiento de plaquetas y factores de la coagulación ${ }^{28}$. Los componentes del sistema de la coagulación tienen un papel en la modulación de la respuesta inflamatoria por lo cual la CID se asocia con mal pronóstico ${ }^{28}$. La sepsis también es una causa importante de trombocitopenia en los neonatos ${ }^{6}$ y una reducción importante en el recuento de plaquetas $\left(<50 \times 10^{9} / \mathrm{L}\right)$ está asociado con incremento en el riesgo de sangrado ${ }^{28}$. La disminución en la función de las plaquetas en los RN pretérmino aumenta aún más el riesgo de sangrado ${ }^{2}$.

Durante la infección, la mielopoyesis en la médula ósea neonatal se disminuye rápidamente, lo que conduce a neutropenia, especialmente común en la SN por gramnegativos, lo que afecta negativamente la inmunidad antimicrobiana ${ }^{2}$. Los neutrófilos neonatales tienen reducida elasticidad en comparación con los neutrófilos en los adultos, lo que asociado a una presión arterial baja aumenta el riesgo de oclusión microvascular ${ }^{2}$. En los neonatos ocurre una agregación irreversible de los neutrófilos en el espacio vascular, lo que lleva a disminución en la diapédesis, depleción de las reservas medulares e incremento del riesgo de problemas de perfusión tisular, llevando finalmente a disfunción orgánica².

Sustancias producidas por otras células inmunes como factores del complemento, citocinas, factores de coagulación y otras, también contribuyen con el desarrollo de $\mathrm{SN}^{2,3}$. La producción de histamina por los mastocitos como respuesta a la infección probablemente también esté asociada con el choque séptico ${ }^{2}$. Se ha visto que los mastocitos neonatales producen cantidades significativamente mayores de histamina en comparación con los mastocitos de adultos, lo que puede estar asociado con la vasodilatación y el desarrollo de choque ${ }^{2}$.

La activación excesiva en respuesta a la infección por medio de los TLR del endotelio puede llevar a dilatación y fuga vascular de líquidos y proteínas, por la acción de varias sustancias como citocinas, quimiocinas, moléculas de adhesión, péptidos vasoactivos como el factor de activación plaquetaria, 
Septiembre - Diciembre

tromboxanos, leucotrienos, óxido nítrico, histamina, bradicinina, prostaglandinas, puede contribuir también con el desarrollo de hipovolemia y por último del choque séptico ${ }^{2,25}$.

Otrosfactores que contribuyen con el establecimiento del choque son la limitación del corazón del neonato para incrementar el volumen latido y la contractilidad, la vaso-regulación periférica anormal, alteraciones metabólicas y nutricionales, que incluyen aumento del gasto energético y el consumo de oxígeno, con una disminución en la función mitocondrial, ocasionada por la hipoxia y la presencia de $\operatorname{ROS}^{2,33}$.

El choque séptico termina llevando a una falla multiorgánica 3 , lo que se relaciona con un pronóstico ominoso, el bajo gasto cardiaco, la falla en la microcirculación, la formación de microtrombos y CID, puede llevar a compromiso de prácticamente todos los órganos, lo que termina por cobrar la vida del $\mathrm{RN}^{2}$.

\section{Biomarcadores en el diagnóstico de sepsis neonatal}

El reto de la identificación de un biomarcador para SN se refleja en el hecho que más de 3000 moléculas han sido propuestas, con cerca de 200 candidatos evaluados ${ }^{34}$. Un biomarcador ideal para SN debe identificar tempranamente y con precisión la enfermedad; la posibilidad de medir el indicador diariamente aumenta la probabilidad de detección temprana, además, un mínimo requerimiento de sangre (se ha sugerido $<0,1 \mathrm{~mL}$ ) es también una característica importante, así como la concordancia en su medición cuando se use sangre capilar o venosa ${ }^{24}$. Debe tener ciertas propiedades como un punto de corte óptimo y estandarizado5, alta sensibilidad y valor predictivo negativo ${ }^{25}$, y una especificidad y valor predictivo positivo $>0,85^{26}$. Los niveles de los biomarcadores se alteran durante el curso de la enfermedad, razón por la que son útiles en el diagnóstico etiológico ${ }^{5}$ y en el seguimiento ${ }^{7}$, además de ser útiles en el monitoreo de la respuesta al tratamiento ${ }^{24}$. Otras características que se buscan en el marcador ideal son la rapidez en la obtención de los resultados, poder ser medido automáticamente, que se realice una medida cuantitativa, que sea accesible y de bajo costo $^{16}$. Los biomarcadores usados se han clasificado en 3 grupos, clásicamente: reactantes de fase aguda, citocinas - quimiocinas y antígenos de superficie; para la presente revisión se incluyó el grupo de "otros marcadores", donde se incluyen algunos marcadores con uso no difundido ampliamente y que han sido estudiados recientemente (Ver Tabla 1). Los biomarcadores en SN también se han clasificado dependiendo en tiempo en que ocurre su expresión en marcadores tempranos (e.g. CD64 o interleucinas) y tardíos (e.g. $\mathrm{PCR}, \mathrm{PCT})^{24}$.

Tabla 1. Biomarcadores de sepsis neonatal

\begin{tabular}{|c|c|}
\hline Reactantes de fase aguda & Citoquinas \\
\hline $\begin{array}{l}\text { Proteína C reactiva (PCR) } \\
\text { Procalcitonina (PCT) } \\
\text { Amiloide sérico } A(A S A) \\
\text { Hepcidina } \\
\text { Lipopolisacárido de unión a proteína (LUP) } \\
\text { Lectina de unión a manosa (LUM) }\end{array}$ & $\begin{array}{l}\text { Interlequina-6 (IL-6) } \\
\text { Interleuquina-8 (IL-8) } \\
\text { Interleuquina-10 (IL-10) } \\
\text { Factor de necrosis tumoral alfa (TNF-a) } \\
\text { CCL5 (RANTES) } \\
\text { CXCR-4 }\end{array}$ \\
\hline Antígenos de superficie celular & Otros biomarcadores \\
\hline $\begin{array}{l}\text { CD11 } \\
\text { Presepsina o CD14 subtipo soluble (CD14Ss) } \\
\text { CD32 } \\
\text { E-selectina (CD62) } \\
\text { L-selectina (CD62L) } \\
\text { CD64 } \\
\text { CD69 } \\
\text { CD163 soluble } \\
\text { Receptor del activador del plasminógeno tipo uroquinasa } \\
\text { soluble (suPAR) } \\
\text { Receptor de activación expresado en células } \\
\text { mielomonocíticas soluble-1 (sTREM-1) } \\
\text { Moléculas de adhesión intercelular-1 (ICAM-1) } \\
\text { Moléculas de adhesión de células vasculares-1 (VCAM-1) }\end{array}$ & $\begin{array}{l}\text { Resistina } \\
\text { Fibronectina } \\
\text { Haptoglobulina } \\
\text { Neopterina } \\
\text { Pentraxina 3 (PTX3) } \\
\text { Lipoproteína Apo-A } \\
\text { Calprotectina } \\
\text { Proadrenomedulina } \\
\text { Marcadores genómicos } \\
\text { Marcadores proteómicos }\end{array}$ \\
\hline
\end{tabular}




\section{Reactantes de fase aguda}

\section{Proteína C reactiva}

La Proteína C Reactiva (PCR) es el reactante de fase aguda más ampliamente estudiado y utilizado en SN, particularmente en SN temprana ${ }^{26}$. Es una proteína de estructura pentamérica ${ }^{5}$ que pertenece a la familia de las pentraxinas ${ }^{19}$, sintetizada en el hígado ${ }^{32}$; se eleva como respuesta a un proceso inflamatorio por el aumento en los niveles de IL-623, IL-1 $\beta$ y TNF- $\alpha^{5}$. Es medida por técnicas de inmunoturbidimetría y nefelometría ${ }^{18}$. Sus niveles se elevan dentro de 6-12 $\mathrm{h}$ desde iniciada la infección, con su pico máximo a las $48 \mathrm{~h}^{16}$. Su vida media es de $19-48 \mathrm{~h}$ y puede aumentar hasta 1000 veces durante una infección 7,35 . Se considera que es eficiente para confirmar 0 descartar la presencia de infección después de 24 $\mathrm{h}$ de sospechado el proceso infeccioso ${ }^{10}$. El punto de corte más preciso y consistente en los reportes es $10 \mathrm{mg} / \mathrm{L}^{11,18,26}$, aunque otros autores proponen $>6$ $\mathrm{mg} / \mathrm{L}$ como el valor aceptado para el punto de corte 5 . Adicionalmente, se están haciendo estudios para comprobar la utilidad de PCR de alta precisión, la cual se considera normal cuando el valor es $<1 \mathrm{mg} / \mathrm{L}$, punto de corte que ha demostrado poder ser más sensible que la prueba convencional en el diagnóstico de SN5. Las mediciones seriales durante las primeras $24-48$ $\mathrm{h}$ tienen mejor sensibilidad (74-98\%) y especificidad (71-94\%); estas mediciones se usan también para evaluar la respuesta a la terapia antibiótica ${ }^{14,26}$. El valor predictivo positivo varía entre $77-100 \%$ y el valor predictivo negativo varía entre $73-98 \%{ }^{18}$. Los valores normales seriados tienen un $99 \% 5$ de valor predictivo negativo para el diagnóstico de infección, por lo que puede ayudar en la decisión de suspender la terapia antibiótica, ${ }^{7,17}$. Se han realizado estudios que muestran que los niveles de PCR medidos en saliva tienen buen rendimiento para el diagnóstico de $\mathrm{SN}$ con punto de corte $3,48 \mathrm{ng} / \mathrm{L}$, la PCR en saliva tuvo una sensibilidad de $94,3 \%$ y especificidad del $80 \%$; en un punto de corte de $3,4 \mathrm{ng} / \mathrm{L}$, tuvo una sensibilidad de $94 \%$ y especificidad de $91,3 \%$ en la predicción de $P C R \geq 10 \mathrm{mg} /$ $L^{19}$. Los niveles persistentemente elevados de PCR deben hacer sospechar la presencia una infección por hongos ${ }^{32}$, tratamiento inadecuado debido a resistencia farmacológica o surgimiento de complicaciones ${ }^{24}$. Diferentes valores para la sensibilidad y especificidad se han publicado con respecto a este marcador, lo que puede explicarse por las variaciones en los estudios en cuanto a la definición de sepsis, metodología de las mediciones, valores de referencia, características de los pacientes, entre otros ${ }^{26}$.
Dentro de las limitaciones de la PCR como biomarcador para infección se encuentran la elevación por procesos inflamatorios no infecciosos como el estrés del parto ${ }^{26}$, exposición a glucocorticoides, RPM, asfixia perinatal o choque, administración de surfactante, hemorragia interventricular, neumotórax, convulsiones y otros $^{5}$. La no disponibilidad de rangos de referencia específicos por edad, el desconocimiento de los cambios en la cinética dependiente del peso al nacer y la edad gestacional7, son algunas de sus limitaciones. La PCR tiene una baja sensibilidad para detectar sepsis temprana debido a la demora en la inducción de su síntesis hepática ${ }^{18}$.

\section{Procalcitoning}

La Procalcitonina (PCT) es la pre-hormona de la calcitonina, secretada normalmente por las células C tiroideas ${ }^{27}$. Es una proteína de 116 aminoácidos con un peso molecular de $14 \mathrm{kDa}^{5}$ con un rol importante en el metabolismo del calcio y como reactante de fase aguda en respuesta a estímulos inflamatorios, especialmente bacterias ${ }^{27}$ y se mide por inmunoturbidimetría generalmente ${ }^{18}$. La PCT en el contexto de un proceso infeccioso de origen bacteriano, también se produce en otros órganos como el hígado ${ }^{27}$, células neuroendocrinas del pulmón y los intestinos y en las células polimorfonucleares de sangre periférica ${ }^{27}$, sustentado en que se ha observado que la PCT también se incrementa en pacientes que han sido sometidos a tiroidectomía ${ }^{36}$. Esta se empieza a sintetizar desde 2-4 h desde el inicio de la infección bacteriana, lo que se refleja en un aumento de sus niveles entre 6-8 h desde el inicio del proceso infeccioso ${ }^{7}$. Tiene su pico máximo a las 18-24 h y su vida media es de $24^{5}-30 h^{7,35}$. Los niveles de PCT incrementan hasta las $24 \mathrm{~h}$ de vida y se mantienen estables durante 2 días $^{36}$. La PCT se eleva y regresa antes a la normalidad comparado con la $\operatorname{PCR}^{23}$ y también es más sensible y específica para identificar infecciones bacterianas en los neonatos ${ }^{9,17}$. Un valor de $0,05 \mathrm{ng} / \mathrm{mL}$ de PCT en suero, prácticamente descarta la presencia de sepsis ${ }^{36}$. En un estudio realizado por Chiesa et al., en el cual se incluyeron 83 neonatos sanos, el valor de PCT más alto al nacimiento fue de $0,7 \mathrm{ng} / \mathrm{mL}^{37}$. Los rangos de referencia para los niveles normales están establecidos por hora de vida, tanto para RN a término y pretérmino ${ }^{36}$. Los niveles de PCT son independientes de la edad gestacional', pero se ha demostrado que en RN con 32 o menos semanas de edad gestacional se afecta por la edad post-natals, el punto de corte 


\section{Septiembre - Diciembre}

más usado es $>2,0 \mathrm{ng} / \mathrm{mL}^{27}$. Su sensibilidad ha sido reportada entre $72-79 \%$ y su especificidad entre 72 $90 \%$ al momento del inicio de los síntomas ${ }^{18}$, aunque otros valores también se han reportado debido a las diferencias metodológicas de los estudios ${ }^{7,26,37}$. Los niveles persistentes o en ascenso sugieren un mal pronóstico de la enfermedad ${ }^{25}$.

Los niveles de PCT pueden elevarse en condiciones no infecciosas como asfixia neonatal, hipoxemia y hemorragia intracraneal7. También existe evidencia que sugiere que la edad gestacional menor a 37 semanas, el muy bajo peso al nacer, un puntaje de APGAR de 4 a los 5 minutos, administración prenatal de antibióticos y el uso de surfactante pulmonar puede aumentar los niveles de PCT en la primera semana de vida ${ }^{36}$.

La precisión de esta prueba varía según si se trata de SN temprana o tardía, aún quedan por establecer umbrales distintos según estas condiciones ${ }^{36}$. Varios investigadores han sugerido que el mejor rendimiento de esta prueba se obtiene al medirla a las $24 \mathrm{~h}$ de vida, además, la toma de 2 muestras, una al momento del nacimiento y otra a las $24 \mathrm{~h}$ de vida, tiene más utilidad clínica que una sola muestra aislada ${ }^{36}$. También se ha demostrado un mejor rendimiento de la prueba en combinación con la $\mathrm{PCR}^{16}$. Últimamente se han realizado estudios evaluando también el uso de una relación $\mathrm{PCT} / \mathrm{PCR}$, que ha mostrado ser útili38.

\section{Amiloide Sérico A}

El amiloide Sérico A (ASA) pertenece al grupo de las apo-lipoproteínas, la cual es sintetizada en el hígado, células endoteliales, monocitos y leiomiocitos 5 . Esta se secreta en respuesta al trauma o infección ${ }^{5}$. Se le atribuyen funciones como quimiotaxis, inmunomodulación y regeneración tisular ${ }^{26}$. Los niveles de ASA dependen de la IL-1, la IL-6 y el TNF- $a^{16}$ y varían con la edad5. Diferencias metodológicas asociadas al establecimiento de puntos de corte que van desde $1-68 \mathrm{mg} / \mathrm{L}$ hace que los estudios sean heterogéneos ${ }^{18}$. Se ha visto que sus niveles incrementan desde 8-24 h después de la infección y hasta 1000 veces durante una infección ${ }^{18}$. En general, tiene una especificidad del $92 \%$ y una sensibilidad del $78 \%{ }^{26}$. Los niveles de ASA tienen una relación inversa con la mortalidad?.
Laproteína de unióna LPS(PUL)esuntipo demolécula de reconocimiento de patrón soluble, sintetizada por los hepatocitos y miocitos, principalmente ${ }^{26}$. Cumple dos funciones, unirse y transportar endotoxinas a las células CD14+ (monocitos) y el reconocimiento de PAMPs de espiroquetas, micobacterias, micoplasmas y bacterias grampositivas ${ }^{26}$. Sus niveles se elevan de 6-8 horas de iniciado el proceso infeccioso5. Tiene una sensibilidad de $94,1 \%$ y una especificidad de $77,8 \%$, un valor predictivo positivo de $88,9 \%$ y un valor predictivo negativo de $87,5 \%$ en un punto de corte de $17,5 \mathrm{mg} / \mathrm{L}^{26}$.

\section{$\underline{\text { Hepcidina }}$}

La hepcidina es un péptido de 25 aminoácidos sintetizado principalmente en el hígado, con un rol crítico en la homeostasis del hierro y los procesos inflamatorios ${ }^{3}$. Esta hace parte de un grupo de moléculas con carga positiva, conocidas como péptidos antimicrobianos, con funciones como regular la respuesta inmune y la apoptosis; además, recientemente se han documentado funciones bactericidas, al insertarse en la membrana de fosfolípidos y causar disrupción, lo que provoca salida del contenido citoplasmático ${ }^{3}$. En el contexto de una infección, la circulación de LPS e IL-6 inducen la expresión de hepcidina por los neutrófilos y macrófagos $^{3}$. Los rangos normales en suero reportados han sido $33-71,3 \mathrm{ng} / \mathrm{mL}$ para los neonatos pretérmino y $55,3-86,8 \mathrm{ng} / \mathrm{mL}$ para los recién nacidos a término ${ }^{26}$. Con un punto de corte de $92,2 \mathrm{ng} / \mathrm{mL}$, la sensibilidad y especificidad fueron de $76 \%$ and $100 \%$, respectivamente ${ }^{26}$. Otros péptidos antimicrobianos como la catelicidina y las defensinas también han sido propuestos como potenciales biomarcadores en sepsis neontal ${ }^{3}$.

Otro reactante de fase aguda que ha sido estudiado como biomarcador de sepsis, aunque en menor medida, es la lectina de unión a manosa ${ }^{26}$.

\section{Citocinas y quimiocinas}

Son pequeñas moléculas con una vida media corta, con un papel central en la respuesta inmune ${ }^{25}$. Las concentraciones de las citocinas varían desde $\rho g / m L$ hasta $\mu \mathrm{g} / \mathrm{mL}^{25}$. En el contexto de SN, los 
cambios en los niveles de las citocinas pueden verse reflejados antes que los cambios en los niveles de los reactantes de fase aguda, sin embargo, los costos de las pruebas manuales y la disponibilidad de los equipos para su medición ${ }^{16}$ representan una barrera en la implementación rutinaria en la práctica clínica de estas pruebas ${ }^{26}$. Las más evaluadas en sepsis neonatal han sido la IL-6, IL-8, IL-10 y TNF- $a$ con resultados aceptables.

\section{$\underline{\text { Interleucina-6 }}$}

La IL-6 es la citocina más estudiada en los neonatos ${ }^{25}$. Es una citocina inducida por el LPS y otros componentes bacterianos, que es producida particularmente por monocitos, células dendríticas, linfocitos $B, T$, células endoteliales y fribroblastos ${ }^{5}$. Esta induce la activación de células $B$ y la secreción de anticuerpos, además de estimular la diferenciación de células T citotóxicas ${ }^{25}$. Sus niveles se elevan antes de que se presenten signos o síntomas de infección. Esta elevación de IL-6 induce el aumento en los niveles de $\mathrm{PCR}^{32}$. Los niveles de IL-6 aumentan dentro de las 2-6 h de iniciada la infección ${ }^{16,25}$, tiene una vida media más corta que la PCR y la $\mathrm{PCT}^{27}$, además, su concentración disminuye hasta volverse indetectable en la mayoría de pacientes con infección dentro de las 24-48 h32, siendo aún más rápida esta disminución cuando se administra tratamiento antibiótico ${ }^{16}$, razones por las que se considera que puede ser de utilidad como marcador temprano de la enfermedad ${ }^{18,25}$. Se ha demostrado que en la SN por hongos hay una elevación sustancial de IL-6 y TNF-a, cuando se compara con $\mathrm{SN}$ de etiología bacteriana ${ }^{30}$, aunque se ha reportado también que la IL-6 se eleva más en la $\mathrm{SN}$ por gérmenes gramnegativos ${ }^{32}$. Se han propuesto varios puntos de corte con un rango que va desde 18 $70 \mathrm{pg} / \mathrm{mL}^{26}$ en plasma. Tiene una sensibilidad de $79 \%$, una especificidad del $84 \% 39,44$, y un valor predictivo negativo de 93-100\%. Se mide por ensayo de inmunoadsorción ligado a enzima ${ }^{18}$. Recientemente se han hecho ensayos con una prueba rápida de IL-6, que tarda una hora, y los resultados fueron similares a la prueba de laboratorio usual en cuanto al rendimiento de detección de SN tardía, pero fue menos sensible para los casos de SN temprana ${ }^{24}$. Al combinar la IL-6 con la PCR aumenta su rendimiento de manera importante ${ }^{7,26}$.

\section{$\underline{\text { Interleucina-8 }}$}

La cinética de la IL-8 es similar a la de la IL-6 ${ }^{16}$. Es producida por monocitos, macrófagos, fibroblastos y células endoteliales ${ }^{5}$. Se eleva dentro de las 2-4 $\mathrm{h}$ de iniciada la infección, con un pico a las $2 \mathrm{~h}^{25}$ y luego una rápida declinación a las $4 h^{5}$. A las 48 hya ha disminuido considerablemente ${ }^{25}$. Se relaciona con la activación y quimiotaxis de los neutrófilos. La sensibilidad y la especificidad de la IL-8 para el diagnóstico de SN varía entre $81-92 \%$ y $70-94 \%$, respectivamente, cuando se usan puntos de cortes de 70-90 $\mathrm{pg} / \mathrm{mL}^{16}$. En los RN con menos de 32 semanas de gestación pueden encontrarse niveles más altos ${ }^{25}$. En combinación con la PCR, PCT o IL-6 su rendimiento aumenta ${ }^{40}$. La desventaja de la medición de la IL-8 en comparación con la IL-6 es su límite de detección, ya que para la IL- 6 es $>0,7 \mathrm{pg} / \mathrm{mL}$, mientras que para la IL- 8 es $>10$ $\mathrm{\rho g} / \mathrm{mL}^{25}$.

\section{Factor de necrosis tumoral-alfa}

EI TNF-a es una citocina proinflamatoria de $17 \mathrm{kDa}$ que resulta del clivaje de una proteína transmembrana homotrímera de $26 \mathrm{kDa}^{26}$, producida principalmente por macrófagos, monocitos y células dendríticas ${ }^{25}$. Frente a la exposición de LPS tiene una rápida elevación desde 30 minutos después del estímulo ${ }^{25}$ y alcanza su pico a los 90 minutos $^{25}$. Tiene una vida media estimada de cerca de 70 minutos $^{25}$. Tiene efectos sobre los vasos produciendo dilatación y aumento de la permeabilidad lo que lleva a que se desarrolle edema, disminución del volumen sanguíneo e hipoproteinemia lo que puede progresar a choque ${ }^{25}$, además junto con la IL-1 $\beta$ pertenecen al grupo de citocinas pirógenas, involucradas en el desarrollo de la fiebre ${ }^{25}$. El estímulo del TNF-a induce la producción de otras citocinas como la IL- 6 e IL$8^{25}$. Los niveles de TNF-a no se afectan por la edad gestacional o posnatal5. Se han estudiado varios puntos de corte para el diagnóstico de $\mathrm{SN}$, desde 0,18-20 $000 \mathrm{pg} / \mathrm{L}$. La sensibilidad y la especificidad han sido de $66-68 \%$ y $76-89 \%$, respectivamente ${ }^{26}$.

\section{Interleucina-10}

La IL-10 es una citocina con un rol anti-inflamatorio importante en la sepsis, regulando la producción de 
Septiembre - Diciembre

citocinas proinflamatorias, especialmente los niveles de TNF- $\alpha$, IL-1, IL-6, IFN- $\gamma$, y GM-CSF, por lo cual tiene un papel importante en la fase del CARS, junto con el TFG- $\beta^{25}$. Es producida por monocitos, macrófagos, células $T$, células $B$ y células $N^{25}$. Se ha reportado que una alta relación de IL-10/TNF se asocia con SN tardía severa ${ }^{25}$. En un punto de corte de $17,3 \mathrm{pg} / \mathrm{mL}$, se ha reportado que la IL-10 tiene una sensibilidad del $92 \%$ y una especificidad del $84 \%$ para el diagnóstico de SN confirmada por cultivo ${ }^{26}$. Se ha demostrado que altos niveles de estas citocinas se asocian con choque séptico en niños ${ }^{25}$. También, se ha observado que en los pacientes con sepsis de origen micótico son los que tienen niveles de IL-10 más altos ${ }^{30}$.

\section{Quimiocinas}

Las quimiocinas son citocinas quimiotácticas que direccionan la migración leucocitaria. Estas se clasifican en subgrupos dependiendo la posición de los dos primeros residuos conservados de cisteína cerca del extremo amino-terminal ${ }^{6}$.

El receptor tipo 4 de a-quimiocina (CXCR-4, también conocido como CD184) es específico para el factor-1 derivado del estroma (SDF-1, también llamado CXCL12), y es una molécula con alta actividad quimiotáctica para los linfocitos. Su expresión se ha visto aumentada en la superficie de los linfocitos circulantes durante un proceso séptico o la estimulación con $\mathrm{LPS}^{6}$. El CXCL12 pertenece al grupo de quimiocinas CXC, está involucrada en procesos de angiogénesis, migración leucocitaria y otros ${ }^{6}$. La utilidad de estos 2 marcadores, especialmente en combinación con la PCR, ha sido demostrada en el diagnóstico de SN tardía ${ }^{6}$. El CCL5 (más comúnmente conocida como RANTES, por su sigla en inglés), es una proteína de $8 \mathrm{kDa}$, con función quimiotáctica en basófilos, eosinófilos y células T. Se ha visto que sus niveles normales disminuyen en los procesos sépticos y que puede ser útil como biomarcador ${ }^{26}$; además, hay datos que sugieren que los niveles disminuidos de CCL5 pueden ayudar a predecir el desarrollo de $\mathrm{CID}^{2}$.

Otras quimiocinas que podrían ser usadas para el diagnóstico de SN son MCP-1 y IP-10 ${ }^{7}$. Hay estudios que muestran que el IP-10 es un biomarcador temprano de infección sensible 2 .
Sepsis neonatal: aspectos fisiopatológicos y biomarcadores

\section{Antígenos de superficie celular}

Al presentarse una respuesta inflamatoria a causa de una infección, se presenta un aumento en la expresión de antígenos leucocitarios?. Estos antígenos pueden ser medidos con bastante precisión mediante citometría de flujo, pudiéndose obtener resultados en menos de $1 \mathrm{~h}$ y usando un volumen de sangre mínimo, de hasta aproximadamente $50 \mu \mathrm{L}$ de sangre total ${ }^{26}$. Algunas moléculas que se expresan en las membranas celulares pueden ser usadas como biomarcadores de infección. Entre estas se encuentran el receptor de la fracción $\mathrm{C}_{3}$ del complemento (CD11 $\beta / C D 18)^{7}$, presepsina o CD14 subtipo soluble (CD14Ss) ${ }^{26}$, CD16, CD32, E-selectina (CD62), L-selectina (CD62L), CD64 ${ }^{16}, \mathrm{CD} 9^{7}, \mathrm{CD} 163$ soluble, el receptor del activador del plasminógeno tipo uroquinasa soluble (suPAR), receptor de activación expresado en células mielomonocíticas soluble-1 (sTREM-1) $)^{18}$, moléculas de adhesión intercelular-1 (ICAM-1) y moléculas de adhesión de células vasculares-1 (VCAM-1 $)^{26}$. El más estudiado ha sido el CD64?

\section{$\underline{\mathrm{CD} 64}$}

También llamado Receptor Fcy I, es un receptor de alta afinidad para las inmunoglobulinas IgG1 e IgG3 expresado en las membranas de los neutrófilos inactivos, en número de aproximadamente 1000 por célula ${ }^{25}$ esta molécula actúa promoviendo la fagocitosis y es expresado frente a una infección bacteriana, los neutrófilos se activan y la expresión de CD64 se puede incrementar hasta 10 veces en un lapso de 4-6 $\mathrm{h}^{25}$. Se ha reportado una sensibilidad entre $75-95 \%$ y una sensibilidad entre $77-88 \%$ al inicio de los síntomas de la SN tardía, requiriendo solo $50 \mu \mathrm{l}$ de sangre y siendo medido usualmente por citometría de flujo.

\section{Presepsina o CD14 subtipo soluble}

La presepsina es una forma soluble truncada del $\mathrm{CD} 14^{26}$. Sus niveles están aumentados en los neonatos con sepsis, aunque no se evidencian grandes diferencias comparado con los pacientes con SIRS de origen no infeccioso. Se ha reportado un punto de corte $>1.39 \mu \mathrm{g} / \mathrm{mL}$ con sensibilidad de $83 \%$ 
y especificidad de $65 \%$, para neonatos con menos de $48 \mathrm{~h}$ de vida, mientras que, para los neonatos con mayor edad, el punto de corte reportado es $>0.59$ $\mu \mathrm{g} / \mathrm{mL}$, con sensibilidad y especificidad de $67 \%$ y $88 \%$, respectivamente ${ }^{26}$.

\section{Otros biomarcadores}

\section{$\underline{\text { Resistina }}$}

La resistina es una hormona descubierta recientemente cuya función está relacionada con la adipogénesis y el desarrollo de resistencia a la insulina ${ }^{23}$. Esta hormona es secretada en los humanos por las células mononucleares, los macrófagos y los neutrófilos ${ }^{23}$. Hay estudios que soportan que la resistina tiene un rol como agente proinflamatorio ${ }^{23}$. Mas aún, se ha reportado que los niveles de resistina se encuentran elevados en los pacientes con SN comparado con controles sin RPM prolongada ni SN y que este aumento también se correlaciona con un incremento en los niveles de IL-623. Sus niveles disminuyen si la madre ha recibido esteroides ${ }^{23}$.

\section{Fibronectina, haptoglobulina y neopterina}

La fibronectina con una especificidad de $96 \%$ y sensibilidad de $63 \%$, la haptoglobulina con especificidad de $95 \%$ y sensibilidad de $67 \%$, y la neopterina con especificidad de $85 \%$ y sensibilidad de $78 \%$ son biomarcadores proteicos que han sido evaluados para ser usados para el diagnóstico de sepsis neonatal?.

Otros biomarcadores que están siendo estudiados como potenciales biomarcadores de infección son: pentraxina 3 (PTX3), lipoproteína Apo-A, calprotectina y proadrenomedulina, entre otros ${ }^{26}$. Recientemente la proteómica, genómica, el análisis post-genómico ${ }^{1}$ y metabolómica se han perfilado como ramas que pueden proveer herramientas útiles en el estudio de la $\mathrm{SN}^{26}$, pero aún están en sus fases iniciales ${ }^{25}$. Por ejemplo, en el caso de los GBS y la $E$. coli, los principales agentes etiológicos de $\mathrm{SN}$, se ha evaluado la caracterización de genes que codifiquen factores de virulencia de estos gérmenes relacionados con el desarrollo de SN. Así mismo, se puede evaluar la presencia de los factores de virulencia en estos microorganismos?

\section{Conclusiones}

La SN aún representa un problema de salud importante alrededor del mundo ya que su diagnóstico sigue siendo problemático, así como tomar la decisión de iniciar o no un tratamiento antibiótico. Se han venido realizando esfuerzos buscando un biomarcador que pueda ayudar a tomar estas decisiones con más seguridad. En el estudio de su fisiopatología se han descrito múltiples factores que se producen dentro del proceso infeccioso que podrían servir como biomarcadores de la enfermedad. Debido al enfoque de la presente revisión, se dejaron de lado los parámetros hematológicos que han sido ampliamente estudiados también como herramientas en el diagnóstico de SN. A pesar de que son múltiples los biomarcadores que se han estudiado en SN, aún no hay disponibilidad de un biomarcador ideal que se pueda usar de manera individual. El mejor rendimiento de los biomarcadores disponibles se obtiene cuando se usan en combinación; hasta ahora se ha recomendado el uso conjunto de un marcador temprano con uno tardío para mejor rendimiento y en mediciones seriadas. Las diferencias en la metodología entre los diferentes estudios hacen difícil establecer un solo punto de corte y rangos de normalidad para ser tomados como referencia en la práctica clínica, por lo que estudios en cada una de las poblaciones son pertinentes para hallar estos valores. Para las definiciones es pertinente adoptar las directrices de las recomendaciones internacionales con el fin de poder comparar los estudios realizados en los distintos escenarios. Las ramas de las ciencias "-ómicas" incipientes podrían en un futuro ayudar con el diagnóstico más eficiente de la SN.

\section{Agradecimientos}

Este trabajo fue financiado por la Universidad Surcolombiana, convocatoria interna para financiar trabajos de grado \#2631, Vicerrectoría de Investigación.

\section{Referencias bibliográficas}

1. Meng Y-X, Liu Q-H, Chen D-H, Meng Y. Pathway cross-talk network analysis identifies critical pathways in neonatal sepsis. Comput Biol Chem. 2017 Jun;68:101-6. 


\section{Septiembre - Diciembre}

2. Wynn JL, Wong HR. Pathophysiology and Treatment of Septic Shock in Neonates. Clin Perinatol. 2010 Jun;37(2):439-79.

3. Ho J, Zhang L, Liu X, Wong SH, Wang MHT, Lau BWM, et al. Pathological Role and Diagnostic Value of Endogenous Host Defense Peptides in Adult and Neonatal Sepsis: a systematic review. Shock. 2017 Jun;47(6):673-9.

4. Singer M, Deutschman CS, Seymour CW, Shankar-Hari M, Annane D, Bauer M, et al. The Third International Consensus Definitions for Sepsis and Septic Shock (Sepsis-3). JAMA. 2016 Feb 23;315(8):801-10.

5. Sharma D, Farahbakhsh N, Shastri S, Sharma P. Biomarkers for diagnosis of neonatal sepsis: a literature review. J. Matern.-Fetal Neonatal Med. 2018 Jun 18;31(12):1646-59.

6. Badr HS, El-Gendy FM, Helwa MA. Serum stromal-derivedfactor-1 (CXCL12) and its alpha chemokine receptor (CXCR4) as biomarkers in neonatal sepsis. J. Matern.-Fetal Neonatal Med. 2018 Aug 18;31(16):2209-15

7. Chauhan N, Tiwari S, Jain U. Potential biomarkers for effective screening of neonatal sepsis infections: an overview. Microb pathog. 2017; 107(Jun):234-242.

8. Jeon JH, Namgung R, Park MS, Park KI, Lee C. Positive maternal C-reactive protein predicts neonatal sepsis. Yonsei Med J. 2014; 55(1):113-7.

9. Cetin O, Aydın ZD, Verit FF, Zebitay AG, Karaman E, Elasan S, et al. Is maternal blood procalcitonin level a reliable predictor for early onset neonatal sepsis in preterm premature rupture of membranes? Gynecol Obstet Invest. 2017; 82(2):163-9.

10. Ayazi P, Mahyar A, Daneshi MM, Jahanihashemi H, Esmailzadehha N, Mosaferirad N. Comparison of serum il-1beta and $\mathrm{c}$ reactive protein levels in early diagnosis and management of neonatal sepsis. Infez Med. 2014; 22(4):296-301.

11. Kan B, Razzaghian HR, Lavoie PM. An immunological perspective on neonatal sepsis. Trends Mol Med. 201; 22(4):290-302.

12. Ceballos CA, Loaiza N, Romero J, Ospina M, Vásquez EM Caracterización de las gestantes tamizadas para streptococcus agalactiae y su relación con sepsis neonatal temprana, en la clínica del prado de Medellín (Colombia), año 2010. Infectio. 2014; 18(2):66-71.

13. Alvarado-Gamarra G, Alcalá-Marcos KM, Abarca-Alfaro DM, Bao-Castro V. Microbiological and therapeutic characteristics of confirmed neonatal sepsis at a hospital in Lima, Peru. Rev Peru Med Exp Salud Publica. 2016, 33(1): 74-82.

14. Mkony MF, Mizinduko MM, Massawe A, Matee M. Management of neonatal sepsis at Muhimbili National Hospital in Dar es Salaam: diagnostic accuracy of $\mathrm{C}$ - reactive protein and newborn scale of sepsis and antimicrobial resistance pattern of etiological bacteria. BMC Pediatr. 2014, 14(1): 293.

15. Freitas FT de M, Romero GAS. Early-onset neonatal sepsis and the implementation of group B streptococcus prophylaxis in a Brazilian maternity hospital: a descriptive study. Brazilian J Infect Dis. 2017, 21(1): 92-7

16. Gilfillan M, Bhandari V. Biomarkers for the diagnosis of neonatal sepsis and necrotizing enterocolitis: Clinical practice guidelines. Early Hum Dev. 2017, 105:25-33.

17. Bedford Russell AR, Kumar R. Early onset neonatal sepsis: diagnostic dilemmas and practical management. Arch Dis Child - Fetal Neonatal Ed. 2014, 100(4): F1- F5.

18. Hedegaard SS, Wisborg K, Hvas AM. Diagnostic utility of biomarkers for neonatal sepsis - a systematic review. Infect Dis (Auckl). 2015 Mar 4;47(3):117-24.

19. Omran A, Maaroof A, Saleh MH, Abdelwahab A. Salivary C-reactive protein, mean platelet volume and neutrophil lymphocyte ratio as diagnostic markers for neonatal sepsis. J Pediatr (Rio J). 2018 Jan;94(1):82-7.

20. Vivas MC, Rengifo ES, Mendieta LMG. Caracterización epidemiológica de pacientes con Sepsis Neonatal en un hospital de la ciudad de Cali (Colombia), 2014. Arch Med. 2017 Dec 6;17(2):225-33.
21. Jaramillo-Bustamante JC, Marín-Agudelo A, Fernández-Laverde M, Bareño-Silva J. Epidemiología de la sepsis en pediatría: primer estudio colombiano multicéntrico. CES Medicina.2019;1(23) :8592.

22. Pan X, Ji Z, Xue J. Percentage of Peripheral CD19+CD24hiCD38hi Regulatory B Cells in Neonatal Sepsis Patients and Its Functional Implication. Med Sci Monit. 2016 Jul 7;22:2374-2378.

23. Aliefendioglu D, Gürsoy T, Çağlayan O, Aktaş A, Ovalı F. Can Resistin be a New Indicator of Neonatal Sepsis? Pediatr Neonatol. 2014; 55(1):53-7.

24. Ng PC, Ma TP, Lam HS. The use of laboratory biomarkers for surveillance, diagnosis and prediction of clinical outcomes in neonatal sepsis and necrotising enterocolitis. Arch Dis Child Fetal Neonatal Ed. 2015; 100(5):F448-52.

25. Machado JR, Soave DF, da Silva MV, de Menezes LB, Etchebehere RM, Monteiro ML, et al. Neonatal sepsis and inflammatory mediators. Mediators Inflamm. 2014; 2014:1-10.

26. Delanghe JR, Speeckaert MM. Translational research and biomarkers in neonatal sepsis. Clin Chim Acta. 2015; 451(Pt A):46-64.

27. Celik HT, Portakal O, Yiğit S, Hascelik G, Korkmaz A, Yurdakök M. Efficacy of new leukocyte parameters versus serum C-reactive protein, procalcitonin, and interleukin-6 in the diagnosis of neonatal sepsis. Pediatr Int. 2016; 58(2):119-25.

28. Kara S, Emeksiz Z, Alioğlu B, Dallar Bilge Y. Effects of neonatal sepsis on thrombocyte tests. J Matern Neonatal Med. 2016; 29(9):1406-8.

29. Nguyen DN, Stensballe A, Lai JC, Jiang P, Brunse A, Li Y, et al. Elevated levels of circulating cell-free DNA and neutrophil proteins are associated with neonatal sepsis and necrotizing enterocolitis in immature mice, pigs and infants. Innate Immun. 2017; 23(6):524-36.

30. Khaertynov KS, Boichuk S V., Khaiboullina SF, Anokhin VA, Andreeva AA, Lombardi VC, et al. Comparative assessment of cytokine pattern in early and late onset of neonatal sepsis. J Immunol Res. 2017; 2017(1):1-8.

31. Dowling DJ, Levy O. Ontogeny of early life immunity. Trends Immunol. 2014;35(7):299-310.

32. Celik IH, Demirel G, Uras N, Oguz ES, Erdeve O, Dilmen U. Función de la concentración sérica de interleucina 6 y proteína C-reactiva para diferenciar la etiología de la septicemia neonatal. Arch Argent Pediatr. 2015;113(6):534-7.

33. Henderson R, Kim S, Lee E. Use of melatonin as adjunctive therapy in neonatal sepsis: A systematic review and metaanalysis. Complement Ther Med. 2018;39(1):131-6.

34. Wang K, Bhandari V, Chepustanova S, Huber G, O'Hara S, O'Hern CS, et al. Which Biomarkers Reveal Neonatal Sepsis?. PLoS One. 2013;8(12): e82700.

35. Pravin-Charles M, Kalaivani R, Venkatesh S, Kali A, Seetha K. Evaluation of procalcitonin as a diagnostic marker in neonatal sepsis. Indian J Pathol Microbiol. 2018;61(1):81.

36. Bell SG. Procalcitonin and Neonatal Sepsis: Is This the Biomarker We Are Looking For? Neonatal Netw. 2017;36(6):380-4.

37. Chiesa C, Pacifico L, Osborn JF, Bonci E, Hofer N, Resch B. Early-Onset Neonatal Sepsis: Still Room for Improvement in Procalcitonin Diagnostic Accuracy Studies. Medicine (Baltimore). 2015;94(30):e1230

38. Hahn W-H, Song J-H, Kim H, Park S. Is procalcitonin to C-reactive protein ratio useful for the detection of late onset neonatal sepsis? J Matern Neonatal Med. 2018;31(6):822-6.

39. $\mathrm{Hu}$ J, DU P, Bei D. [Diagnostic value of interleukin 6 for neonatal sepsis: a Meta analysis]. Zhongguo Dang Dai Er Ke Za Zhi. 2015;17(11):1176-82.

40. Zhao F, Liu G, Zhang J. [Value of IL-6 and IL-8 in the diagnosis of neonatal sepsis]. Zhongguo Dang Dai Er Ke Za Zhi. 2015;17(12):1311-5. 